\title{
IEEJ Electronics, Information and Systems Society
}

\section{Board of Society <Board Member >}

President

Keiichiro Yasuda, Tokyo Metropolitan Univ.

President Elect

Akira Fukumoto, Toshiba Corp.

Vice Presidents

Yoshizumi Serizawa, CRIEPI

Yasuhiko Jimbo, The Univ. of Tokyo

Officers, Planning \& General Affairs

Takahiro Koharagi,Tokyo Electric Power Company

Naoki Hara, Fujitsu Laboratories Ltd.

Officers, Treasurer

Osamu Shimohira, NEC Corp.

Yasuko Fukuzawa, Hitachi, Ltd.

Officers, Editorial Affairs

Yoshio Izui, Mitsubishi Electric Corp.

Katsutoshi Saeki, Nihon Univ.

Officers, R\&D Management

Naruhiko Mukai, Toshiba Corp.

Kunihiko Oura, Kokushikan Univ.

Officers, Public Information

Seigo Sasaki, National Defense Academy of Japan

Tomoki Hamagami, Yokohama National Univ.

Auditors

Tetsuro Matsui, Fuji Electric Co., Ltd.

Hitoshi Iima, Kyoto Institute of Technology

Secretaries

Shigeru Fujimura, Waseda Univ.

Takashi Itoh, Fujitsu Laboratories Ltd.

Masashi Kitayama, Mitsubishi Electric Corp.

Masayuki Kuribara, Denryoku Computing Center

Makoto Motoki, Kanto Gakuin Univ.

Hiroyuki Oohashi, Toshiba Corp.

Takahide Sato, Univ. of Yamanashi

Masayuki Yoshida, Hitachi, Ltd.

\section{$R \& D$ Steering Committee \\ Chairperson}

Yoshizumi Serizawa, CRIEPI

Vice Chairpersons

Naruhiko Mukai, Toshiba Corp.

Kunihiko Oura, Kokushikan Univ.

Members

Shigeru Fujimura, Waseda Univ.

Nobuo Haneji, Yokohama National Univ.

Yoshiaki Hasegawa, Toshiba Corp.

Fukashi Kamikawa, NEC Corp.

Tomoo Katauri, Toshiba Corp.

Takeyoshi Kato, Nagoya Univ.

Norihisa Komoda, Osaka Univ.

Hiroshi Kumagai, Osaka City Univ.

Shiro Masuda, Tokyo Metropolitan Univ.

Kazuhiro Matsuda, NTT

Kazuyuki Mori, Mitsubishi Electric Corp.

Tadashi Okabe, Tokyo Electric Power Company

Osamu Ono, Meiji Univ.

Yoshifumi Sekine, Nihon Univ.

Shigehito Shimada, Toshiba Corp.

Takashi Shinohe, Toshiba Corp.

Masakazu Suzuki, Tokai Univ.

Shigetaka Takagi, Tokyo Institute of Technology

Haruo Takeda, Hitachi, Ltd.

Akinori Ueno, Tokyo Denki Univ.

Secretary

Hiroyuki Oohashi, Toshiba Corp.

\author{
Editorial Committee \\ Chairperson \\ Yasuhiko Jimbo, The Univ. of Tokyo \\ Vice Chairpersons \\ Yoshio Izui, Mitsubishi Electric Corp. \\ Katsutoshi Saeki, Nihon Univ.
}

\section{Members}

Mingcong Deng, Tokyo Univ. of Agriculture and Technology

Tetsuya Goto, Tohoku Univ.

Hiroto Hamane, Kougakuin Univ.

Takeo Hariu, NTT

Koichi Hidaka, Tokyo Denki Univ.

Hitoshi Iima, Kyoto Institute of Technology

Keiji Iramina, Kyushu Univ.

Hiroyasu Ishikawa, Shibaura Institute of Technology

Tomonori Izumi, Tokyo Denki Univ.

Masashi Kitayama, Mitsubishi Electric Corp.

Kazuaki Masuda, Kanagawa Univ.

Kohji Masuda, Tokyo Univ. of Agriculture and Technology

Fujihiko Matsumoto, National Defense Academy of Japan

Shinichiro Morimoto, AIST

Masayoshi Nakamoto, Hiroshima Univ.

Masaki Nakamura, TEPCO SYSTEMS CORP.

Takahide Sato, Univ. of Yamanashi

Kazuhiro Shouno, Univ. of Tsukuba

Makoto Suzuki, Shonan Institute of Technology

Seiichi Suzuki, Seikei Univ.

Yoji Taniguchi, Hitachi, Ltd.

Masahiko Tanimoto, Mitsubishi Electric Corp.

Isao Tazawa, Hitachi, Ltd.

Kenji Terada, Tokushima Univ.

Secretaries

Yasushi Fujimoto, Osaka Univ.

Naoki Mori, Osaka Prefecture Univ.

\section{Program Committee \\ Group C1 \\ Chairperson \\ Kazuhiro Shouno, Univ. of Tsukuba}

Vice Chairperson

Fujihiko Matsumoto, National Defense Academy of Japan

Secretaries

Yasushi Fujimoto, Osaka Univ.

Tetsuya Goto, Tohoku Univ.

Takeo Hariu, NTT

Keiji Iramina, Kyushu Univ.

Hiroyasu Ishikawa, Shibaura Institute of Technology

Kohji Masuda, Tokyo Univ. of Agriculture and Technology

Seiichi Suzuki, Seikei Univ.

Group C2

Chairperson

Kenji Terada, Tokushima Univ.

Vice Chairperson

Hiroto Hamane, Kougakuin Univ.

Secretaries

Mingcong Deng, Tokyo Univ. of Agriculture and Technology

Hitoshi Iima, Kyoto Institute of Technology

Tomonori Izumi, Tokyo Denki Univ.

Naoki Mori, Osaka Prefecture Univ.

Masayoshi Nakamoto, Hiroshima Univ.

Makoto Suzuki, Shonan Institute of Technology

Yoji Taniguchi, Hitachi, Ltd.

Masahiko Tanimoto, Mitsubishi Electric Corp. 